\title{
Antibiotic Resistance Patterns in Pseudomonas aeruginosa Strains Isolated from Patients Hospitalized in Different Hospital Wards
}

\author{
Tanaz Zabihi ${ }^{1 *}$, Maedeh Shahsanaei Goneirani ${ }^{1}$ \\ ${ }^{1}$ Department of Microbiology, Taligene Pars Company, Isfahan Science and Technology Town, Isfahan, Iran
}

Corresponding Author: Tanaz Zabihi, MSc in Microbiology, Department of Microbiology, Taligene Pars Company, Isfahan Science and Technology Town, Isfahan, Iran. Tel: +98-3142646414, E-mail: zabihitanaz@yahoo.com

Received May 24, 2017; Accepted June 12, 2017; Online Published June 29, 2017

\begin{abstract}
The discovery of antibiotics followed by the extensive production of new antibiotics and their widespread use in the treatment of bacterial infectious diseases has led to bacterial resistance to antibacterial agents. Despite global efforts to improve the status of antimicrobial treatment, the phenomenon of resistance against gram-negative bacteria has increased in communities and hospitals. Pseudomonas aeruginosa, the most common human pathogen, is an oxidase-positive and glucose non-fermentative gram-negative bacillus considered an important opportunistic pathogen and the cause of mortality in patients with cystic fibrosis, neoplasms, and severe burns. Infection caused by Pseudomonas aeruginosa is one of the most important complications in hospitalized patients, and its incidence is increasing significantly in developed and developing countries. Based on the literature, it can be concluded that antibiotic resistance is higher in developing countries than developed ones, which is likely due to excessive antibiotic use in developing countries or the control of antibiotic use in developed ones.

Keywords: Pseudomonas Aeruginosa; Antibiotic Resistance; Iran

Citation: Zabihi T, Shahsanaei Goneirani M. Antibiotic resistance patterns in Pseudomonas aeruginosa strains isolated from patients hospitalized in different hospital wards. Int J Med Rev. 2017;4(2):40-42. doi: 10.29252/ijmr-040202.
\end{abstract}

\section{Introduction}

The Pseudomonas genus is a complex of nonfermentative aerobic gram-negative bacteria commonly found in soil, water, and plants and often found in small numbers in the natural intestinal flora and skin of humans; it is considered the main pathogen in this group. Pseudomonas pathogens rarely cause disease and are categorized based on RNA/DNA similarity and conventional planting characteristics. Pseudomonas aeruginosa is widely distributed in nature and is often found in wet hospital environments. These bacteria can be colonized in healthy humans, acting as a saprophyte. ${ }^{1}$ Several species of this genus cause diseases in animals and plants. Most Pseudomonas microorganisms do not infect humans, but some of them are potentially important pathogens for hosts with impaired immune systems. Usually human infections are severe and difficult to treat because of resistance to different conventional antibiotics and weak host conditions. Pseudomonas aeruginosa is the most common human pathogen in the pseudomonas genus. Pseudomonas aeruginosa is the third most common cause of nosocomial infections after Staphylococcus aureus and Escherichia coli in some hospitals. These bacteria is a major pathogen and the leading cause of mortality in patients with cystic fibrosis, neoplasms, and severe burns. $^{2}$ In recent years, drug resistance has significantly increased in different pathogenic bacteria and has made the treatment of infections difficult. Therefore, there is a growing need for safe and effective drugs for use in treating drug-resistant infections. ${ }^{3} \quad$ Pseudomonas aeruginosa is naturally resistant to several antibiotics and can be resistant to antibiotics such as penicillin, ceftazidime, ciprofloxacin, aminoglycosides, and carbapenems. The prevalence of antibiotic resistance is increasing; more than $10 \%$ of strains isolated across the world are resistant to multiple drugs. ${ }^{4}$

An Introduction to Drug Resistance

Resistance to antimicrobial agents and antibiotics among human pathogens has now entered the phase of multiple drug resistance (MDR). Despite the presence of numerous antibiotics and pharmaceutical factories and billions of dollars spent, the elimination of bacterial

Copyright (C) 2017 The Author(s). This is an open-access article distributed under the terms of the Creative Commons Attribution License (http://creativecommons.org/licenses/by/4.0), which permits unrestricted use, distribution, and reproduction in any medium, provided the original work is properly cited. 
diseases is still far from mind, mainly due to the presence of numerous bacteria that resist the antibiotic effects. It is worth noting that for antibiotics to function better, how these substances act in the body should be considered. First, antibiotics should identify their target. Next, they must reach their proper concentration after reaching the target, and then they must maintain that concentration for a sufficient period of time. Some treatment problems are caused by the antibiotic's inability to selectively reach the target, cross the blood brain barrier, or reach the bacteria present in an abscess. The concentration of the antibiotic is also important. In some cases, it has been observed that antibiotics are rapidly excreted from or metabolized in the body, and this reduces the serum concentration of antibiotic, resulting in the ineffective treatment of bacterial infections that require large amounts of drug and prolonged contact with antimicrobial agents for elimination. In some cases, however, the antibiotic concentration may increase rapidly in a particular organ or specific tissue, while infectious parts may remain out of its reach. The mechanism of antibiotic resistance can be genetic or non-genetic. Today, in addition to trying to produce semi-synthetic antibiotics from existing antibiotics to be effective against resistant strains, attempts have been made to extend the recommendations for the proper use of antibiotics as much as possible in order to prevent the emergence of resistant strains. ${ }^{5}$

\section{Multidrug Resistance}

Many bacteria are resistant to several classes of antibiotics (at least three different classes) and use various strategies to overcome the stress induced by an antibiotic's chemotherapeutic effect. These bacteria are called MDR, which are a major concern today in hospitals and other medical centers. MDR strains of Pseudomonas aeruginosa have been shown to exist in hospitals, isolated from the environment and hands of the personnel. Studies in Iran have shown that the multiple drug resistance of Pseudomonas aeruginosa strains has reached a worrying level in nosocomial infections. ${ }^{6}$ The main mechanism of resistance in these bacteria is the active transfer of drugs from the cell to the environment through pumps that send out a wide range of compounds harmful to bacteria, such as antibiotics. Additionally, the mutation of antibiotic target sites or the synthesis of enzymes changing the drug can induce MDR resistance in bacteria. In all of these cases, bacterial strains carrying resistive factors are selected through the use of antimicrobial molecules, because sensitive strains are eliminated, while resistant strains grow and survive. ${ }^{7}$

\section{Treatment of Infections Caused by Pseudomonas aeruginosa Bacteria}

One of the most important causes of nosocomial infections is the definite spread of drug resistance. ${ }^{8}$ Resistance to antimicrobial agents among numerous pathogens, especially pathogens affecting nosocomial infections that impair the treatment of infections, has extended globally, leading to increased mortality rates in patients in different hospital wards. ${ }^{9}$ Pseudomonas aeruginosa is the most common human pathogen of the pseudomonas genus and the third most common cause of nosocomial infections after Staphylococcus aureus and Escherichia coli, which leads to mortality in patients suffering from cystic fibrosis, neoplasms, and severe burns. $^{5}$ The term "nosocomial infection" refers to a serious disease unrelated to the underlying disease of the person seeking treatment in a hospital. ${ }^{6}$ Treatment for pseudomonas infection is difficult, and treatment with appropriate drugs is not always successful. Pseudomonas aeruginosa infections should not be treated with one drug, as resistant strains develop quickly. Penicillins effective against these bacteria include ticarcinin, mesocillin and piperacillin, usually prescribed with an aminoglycoside such as gentamicin, tobramycin or amikacin. Other drugs effective against pseudomonas aeruginosa include aztreonam, imipenem, new quinolones including ciprofloxacin, and new generation cephalosporins such as cefaprazone. Ceftazidime is also used in the initial treatment of pseudomonas aeruginosa infections. $^{5}$

\section{Discussion}

Antibiotic resistance to gram-negative bacilli, especially enterobacteriaceae, pseudomonas aeruginosa, and acinetobacter, has become a growing problem around the world. Resistant bacteria can significantly impede the treatment maintenance. ${ }^{9}$ Several studies have addressed antibiotic resistance in different countries, most of which suggest using microbial and molecular techniques together as reliable and documented tools. Antibiotic resistance in aztreonam was $1 \%$ in France, $48.8 \%$ in Russia, $11.9 \%$ in the United States (USA), 23\% in Spain, and $66.7 \%$ in Isfahan, Iran. ${ }^{7,10-13}$ Antibiotic resistance to tobramycin has been reported as $25 \%$ in France, $5.4 \%$ in the USA, $10 \%$ in Spain, and $91.7 \%$ in Isfahan, Iran, which is alarmingly high; in other words, it is possible that in the near future, no other antibiotic will be effective against pseudomonas infections in Isfahan. $7,10,12,13$ The antibiotic resistance to ticarcillin was 38\% in France and $64 \%$ in Canada. According to the results of the present study, the resistance to this antibiotic was $83.3 \%$ in Isfahan, which is significantly higher than the abovementioned regions and indicates the low effect of this antibiotic in Isfahan, possibly due to the wide use of this antibiotic in treatment regimens. ${ }^{13,14}$ The antibiotic resistance to ceftazidime was reported as 9\% in France, $12.3 \%$ in Brazil, $26 \%$ in Turkey, $4.6 \%$ in Japan, $35 \%$ in Russia, $12 \%$ in Canada, $11.1 \%$ in the USA, $15 \%$ in Spain, and $75 \%$ in Isfahan. $7,10,11,15,16$ Resistance to imipenem was $18.5 \%$ in France and $3.8 \%$ in Japan. ${ }^{12,16}$

\section{Conclusion}

According to the results of studies, it can be concluded that antibiotic resistance is higher in developing countries than developed countries, possibly due to the 
excessive use of antibiotics in developing countries or the control of antibiotic use in developed countries.

\section{Acknowledgments}

The authors express their sincere appreciation for the cooperation of the personnel at Taligene Pars Co. who assisted in this research.

\section{References}

1. Lyczak JB, Cannon CL, Pier GB. Establishment of Pseudomonas aeruginosa infection: lessons from a versatile opportunist. Microbes Infect. 2000;2(9):1051-60. pmid: 10967285.

2. Wolfang C. Zincase Microbiology. 4th ed. Tehran: Aijiz; 2010.

3. Bhat G, Suman E, Shetty A, Hegde B. A Study on the ASAP nano-silver solution on pathogenic bacteria and Candida. J Indian Acad Clin Med. 2009;10(2):15-7.

4. Fazeli H, Havaei SA, Solgi H, Shokri D, Motallebirad T. Pattern of Antibiotic Resistance in Pesudomonas Aeruginosa Isolated from Intensive Care Unit, Isfahan, Iran. J Isfahan Med Sch. 2013;31(232):433-8.

5. Malekzada F. General Microbiology. 2nd ed. Tehran: Tehran University of Medical Sciences; 2005.

6. Fazeli H, Moslehi Z, Irajian G, Salehi M. Determination of Drug resistance patterns and detection of bla-VIM gene in Pseudomonas aeruginosastrains Isolated from burned patients in the Emam Mosa Kazem hospital, Esfahan, Iran (2008-9). Iran J Med Microbiol. 2010;3(4):1-8.

7. Zabihi T, Karbasizade V, Ahadi AM. Detection of blakpc genes in Pseudomonas aeruginosa Isolated from clinical samples. Isfahan, Iran: Islamic Azad University Falavarjan Branch; 2014.

8. Liberati A, D'Amico R, Pifferi, Torri V, Brazzi L. Antibiotic prophylaxis to reduce respiratory tract infections and mortality in adults receiving intensive care. Cochrane Database Syst Rev. 2004(1):CD000022. doi: 10.1002/14651858.CD000022.pub2. pmid: 14973945.

9. Hrabak J, Walkova R, Studentova V, Chudackova E, Bergerova T. Carbapenemase activity detection by matrixassisted laser desorption ionization-time of flight mass

\section{Authors' Contributions}

TZ and MSG contributed equally to this research.

\section{Conflict of Interest Disclosure}

The authors declare that they have no conflicts of interest.

Funding

None.

spectrometry. J Clin Microbiol. 2011;49(9):3222-7. doi: 10.1128/JCM.00984-11. pmid: 21775535.

10. Shawar RM, MacLeod DL, Garber RL, Burns JL, Stapp JR, Clausen CR, et al. Activities of tobramycin and six other antibiotics against Pseudomonas aeruginosa isolates from patients with cystic fibrosis. Antimicrob Agents Chemother. 1999;43(12):2877-80. pmid: 10582875.

11. Bouza E, Garcia-Garrote F, Cercenado E, Marin M, Diaz MS. Pseudomonas aeruginosa: a survey of resistance in 136 hospitals in Spain. The Spanish Pseudomonas aeruginosa Study Group. Antimicrob Agents Chemother. 1999;43(4):981-2. pmid: 10103217.

12. Cavallo J, Fabre R, Leblanc F, Nicolas-Chanoine M, Thabaut A. Antibiotic susceptibility and mechanisms of $\beta$-lactam resistance in 1310 strains of Pseudomonas aeruginosa: a French multicentre study (1996). J Antimicrob Chemotherap. 2000;46(1):133-6. doi: 10.1093/jac/46.1.133.

13. Karakoc B, Gerceker AA. In-vitro activities of various antibiotics, alone and in combination with amikacin against Pseudomonas aeruginosa. Int J Antimicrob Agents. 2001;18(6):567-70. pmid: 11738346.

14. Sivolodskii EP. [Antibiotics sensitivity and characteristics of the esculin-positive Pseudomonas aeruginosa biovar]. Antibiot Khimioter. 2000;45(8):17-20. pmid: 10989719.

15. Kato K, Iwai S, Kumasaka K, Horikoshi A, Inada S, Inamatsu $\mathrm{T}$, et al. Survey of antibiotic resistance in Pseudomonas aeruginosa by The Tokyo Johoku Association of Pseudomonas Studies. J Infect Chemother. 2001;7(4):25862. doi: 10.1007/s101560100047. pmid: 11810595.

16. Zabihi T, Karbasizade V, Ahadi AM. Comparative Analysis of Three Methods for Determination of Imipenem Resistance in Pseudomonas aeruginosa. J Med Bacteriol. 2015;4(6):3741. 